\title{
Research on the impact of Chinese local government patent funding on the development of regional patent
}

\author{
Zhigang Ji ${ }^{*}$, and Xinkai Dong \\ School of Intellectual Property, Nanjing University of Science and Technology, Nanjing, China
}

\begin{abstract}
This paper aims to study the impact of Chinese patent funding support on the development of regional patent layout.Data samples were selected from Guangxi, Jiangsu and non-pilot provinces, and data samples were selected from the period 2015-2018.This paper discusses the influence relationship by game theory. The conclusion is that the support of patent funding has a positive impact on economically developed areas and a negative impact on economically less developed areas.Other measures are required to eliminate the aforementioned negative effects.
\end{abstract}

Keywords: Patent grants, Patent layout, Risk game, Innovative methods, Long-term mechanism.

\section{Introduction}

The support of government patent funded funds plays a significant positive and effective role on the development of regional patent layout (Mao Hao, 2015), However, the disadvantages of patent funding for the development of regional patent layout have been shown, highlighted in the heavy application and light application (Zhang Xiaoyue et al., 2017). Liu Huifeng, Liu Xuefeng, the quality of the patent was discussed and studied from the perspective of patent funding. Field experts from the above three perspectives to discuss. The research methods used in the paper are mainly mathematical models and algorithms for association influence, but also directly inferred.

Therefore, the research significance of this article is based on the pilot work of national intellectual property work, making data analysis and theoretical discussion for the pilot work, and also playing a useful reference for the future demonstration work of national intellectual property regional patent layout.

Patent data analysis from 2015-2018 in the three pilot provinces

Please follow these instructions as carefully as possible so all articles within a conference have the same style to the title page. This paragraph follows a section title so it should not be indented.

\footnotetext{
*Corresponding author: 51583297@qq.com
} 


\subsection{Guangxi province patent data}

Guangxi Province Patent Data 2015-2018 (Retrieval string "aa:(Guangxi) AND (ad: [201t 0101 TO 201t 1231])", $t=5,6,7,8$. Retrieved in October 2020).

It can be found that from 2015 to 2018, the general patent data of Guangxi Province were: 38566 in 2015,51119 in 2016,50502 in 2017 and 35918 in 2018, presenting " $\eta$ ". The data of roughly the same development curve as the total statistical data is the number of invention disclosure patents during the four years, and the number of invention disclosure patents is $23,484,34,669,33,429$ and 19,095 , respectively. During the four years, three types of data achieved a positive growth trend, namely the utility model authorization, design authorization and WIPO data, among which the utility model authorization changes were $7650,8823,11807,12243$ respectively, and the design authorization change was $2644,3310,3807,4461$ respectively. WIPO data, 30,38,73,87, from 2015-2018, respectively. The data in the figure shows that during the pilot in 2016-2017, the invention patent data in 2015,2018 data is falling to the total before the pilot year, which shows that Guangxi regional intellectual property layout only in the utility model, design the two low innovation value categories achieved positive growth, the only gratifying is the growth of WIPO channel registered international patent data in the four years.

\subsection{Jiangsu province patent data}

Jiangsu Province Patent Data 2015-2018 (Retrieval string "aa:(Jiangsu) AND (ad: [201t 0101 TO 201t 1231])", $\mathrm{t}=5,6,7,8$. Retrieved in October 2020).

It can be found that from 2015 to 2018 , the general situation of the patent data in Jiangsu Province was: 338,000 in 2015,373,000 in 2016,449,000 in 2017 and 450,000 in 2018 , showing the overall significant growth during the pilot period and a positive growth decline after the pilot period. The data with the same development curve as the total statistical data are the number of new patents, 146,000,189,000 and 218,000 respectively and invention patents, 130,000,150,000,189,000 and 181,000 respectively. During the four years, only the appearance of the design data continued to show a negative growth trend.

\subsection{Hebei province patent data}

Hebei Province (2015-WIPO Pilot Province) (2018 Patent Data (Retrieval string "aa:(Hebei) AND (ad: [201t 0101 TO 201t 1231])", $\mathrm{t}=5,6,7,8$. Retrieved time in October, 2019).

It can be found that from 2015 to 2018 , the general situation of the patent data in Hebei Province was: 42,641 in 2015,52,107 in 2016,64,115 in 2017, and 73,212 in 2018, showing the rapid growth during the overall presentation period.Each data and the total statistical data basically show the same development curve.

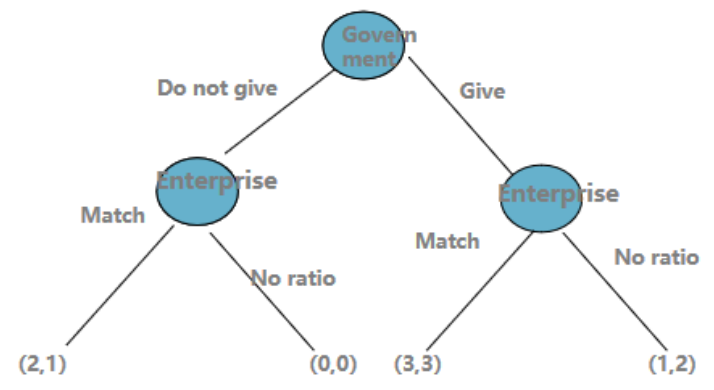

Fig.1. Game tree between government-guided funds and the regional patent layout of enterprise investment. 


\section{Risk game analysis of the patent layout of government-guided funds}

Assuming that the fluctuation phenomenon of patent application data in Guangxi Province is caused by the guidance of the national regional patent layout pilot funds, how can we make this data show an increasing fluctuation such as Jiangsu or Hebei Province by establishing a game model? And makes the increasing fluctuation more optimized compared with Hebei.

Participants: Government, regional enterprises

Strategy: Give funds; (ratio, mismatch)

If the government budget gives enterprise funds for regional enterprise patent layout, the government can get 2 units of income, consuming one unit, and the enterprise gets 2 units of income; enterprise matching funds for patent layout, requiring one unit, one unit of income, and the government gets 2 units of income. See Figure 1-The game tree between government-guided funds and enterprise investment in regional patent layout.

According to the above equilibrium results: give funds, ratio. Equilibrium strategy: to give funds, (ratio, not ratio), payment: $(3,3)$, the government first does not give, commitment to the enterprise ratio after the subsidy, pay $(2,1)-\cdots(3,3)$ is the optimal strategy.

Therefore, it can be concluded that 1: government financial support is an important driving force of regional patent layout. The balance in the model is funds first and the ratio of enterprises, but this balance is often difficult to confirm the quality and sustainability of patent layout. Therefore, we can see that more and more government support will support the use of post-subsidies, which is relatively better suitable for high-quality regional patent layout scenarios. That is, the government does not give, promise the ratio of enterprise subsidy is the optimal way. Conclusion 2: A third party can be considered to support regional patent layout to realize a long-term mechanism, and government funds are gradually replaced. Conclusion 3: Introduce insurance mechanism to support regional patent layout and realize long-term mechanism, and government funds are gradually replaced. Conclusion 4: Government funds can be considered as venture capital funds to support regional patent layout to realize the value of government funds.

\section{Patent data analysis from $\mathbf{2 0 1 5 - 2 0 1 8}$ in Guangxi, Jiangsu and Hebei provinces}

Patent data from 2015 to 2018 from Guangxi, Jiangsu and Hebei provinces were obtained through the website database of the State Intellectual Property Office of the People's Republic of China, including the following variables: Total patents $(10,000)$; Total invention patents (ten thousand pieces); Total new patents $(10,000)$; Total appearance design (10,000 pieces); number of counties (individual); Government funding accounts for an incremental patent input ratio (\%).

In the evaluation index system, there are many observed data, so there are inevitable multiple collinearity problems between the indicators, so it is necessary to calculate the correlation matrix of the observed data.

Styl According to the correlation coefficient matrix in Table 1, the total number of patents $(10,000)$ is highly positively correlated with total invention patents $(10,000)$ and total new patents $(10,000)$, and the total patents $(10,000)$ are strongly positively correlated with the proportion of government funds in incremental patent investment (\%). To eliminate the correlations between the metrics, the factors were extracted by factor analysis.

Suitability test: KMO test and Bartlett test are the premise of factor analysis, confirming whether the analyzed variables are suitable for factor analysis, KMO test and Bartlett test 
for 6 index variables. Results: KMO test result $0.7753996>0.5$, indicating that data are more suitable for factor analysis and $\mathrm{P}$ value is $1.313353 \mathrm{e}-38<0.050$, indicating that data are suitable for factor analysis.

Calculates the factor score: According to the factor score formula, you can get the factor score table of the three provinces, municipalities and municipalities directly under the Central Government, and take the variance contribution rate of the three factors as the weight number to find the comprehensive score of the three provinces in China, and then sort. The results are detailed in Table 2 .

The comprehensive score of 3 provinces basically explains the comprehensive strength of the intellectual property layout, among which the comprehensive score is above the average level of 3 provinces; while the region with negative comprehensive score shows that its comprehensive strength is below the average level of 3 provinces.

Table 1. Correlation matrix.

\begin{tabular}{c|c|cccccc}
\hline & $\mathbf{x 1}$ & $\mathbf{x 2}$ & $\mathbf{x 3}$ & $\mathbf{x 4}$ & $\mathbf{x 5}$ & $\mathbf{x 6}$ \\
\hline $\mathbf{x 1}$ & 1 & 0.7816825 & 0.9897972 & 0.55807749 & 0.8591197 & 0.4264471 \\
$\mathbf{x 2}$ & 0.7816825 & 1 & 0.7660047 & 0.08494884 & 0.4903701 & 0.367323 \\
$\mathbf{x 3}$ & 0.9897972 & 0.76600468 & 1 & 0.59143856 & 0.8625935 & 0.4170458 \\
$\mathbf{x 4}$ & 0.5580775 & 0.08494884 & 0.5914386 & 1 & 0.7147545 & 0.1937758 \\
$\mathbf{x 5}$ & 0.8591197 & 0.49037012 & 0.8625935 & 0.71475447 & 1 & 0.4684224 \\
$\mathbf{x 6}$ & 0.4264471 & 0.36732305 & 0.4170458 & 0.19377583 & 0.4684224 & 1 \\
\hline
\end{tabular}

\section{Extended thinking of risk game and data statistical analysis from government-guided funds in regional patent layout}

Through the front of Guangxi, Jiangsu and Hebei provinces regional patent layout data analysis and government guide funds in regional patent layout risk game and data statistical analysis, Jiangsu province as at the same time to carry out the intellectual property regional layout pilot province, abundant innovation resources, full use, government funds play a good role in guiding intellectual property related resources reasonable layout. As an underdeveloped province with intellectual property rights, Hebei Province can also make full use of limited innovation resources and government resources to steadily promote the development of intellectual property rights, especially the patent layout, and can maintain a relatively balanced and stable development of all types of patents. Compared with the first two provinces, Guangxi province is not only underdeveloped intellectual property provinces, as the first national intellectual property pilot provinces, the pilot around 2016 and 2017, patent layout and development, as a patent type of invention patent data in 2016 in 2015,2017 and 2018 data fell year by year under the pilot year, which shows that the patent layout work has not played a good positive role. The regional intellectual property layout in Guangxi has only achieved positive growth in the two low innovation value categories, utility models and appearance design.

According to the Opinions of the General Office of the State Council on County Innovation-Driven Development and combined with the previous analysis, this article puts forward targeted promotion measures for the development and layout of regional intellectual property rights. 


\subsection{Innovation investment:}

Set up a private joint fund of science and technology investment government + enterprises;

sets up a science and technology investment mode (including regional industry, university, research and + capital) based on blockchain technology regional advantages and advantages of advanced technology patent layout. The main technical support of blockchain technology is the application of +TRIZ theory and evolution law of + patent intelligence analysis technology;

Implement the macro plan for regional scientific and technological innovation, especially the establishment of the municipal-level regional innovation and application capacity leap demonstration zone of "one central + , seven demonstration zones and counties";

Regularly and irregularly trains and promotes the basic knowledge and techniques of innovation methods and students of enterprise engineers, technical principals, social innovation and entrepreneurship personnel, and teachers and students in regional schools, so as to realize the benign operation of regional innovation ecosystem. Improve the independent innovation and application ability of enterprises in product design and production links, improve the quality of enterprise engineer innovation, promote the overall improvement of regional enterprise gradual innovation ability, promote the existing product technology upgrading and production process optimization of regional enterprises, and realize the increase of technological innovation-guided research and development investment of enterprises.

\subsection{Innovation output}

Implements the three-dimensional construction of regional innovative application capability system; focuses on promoting the promotion of basic education of innovation methods, improving the quality of technological achievements, and thus increasing the proportion of invention authorization; building the patent protection layout and the patent protection pool and patent protection pool.

Enterprise publicity: policy publicity to the enterprise management and technical level; registered enterprises shall guide and explain under the Science and Technology Bureau according to the industry.

Short-term mechanism: invention patent transfer, immediate. By contacting the enterprise, understanding the needs of the enterprise, market docking the patent technology urgently needed by the enterprise, and purchase the patent right from the patents that universities, enterprises or individuals have authorized or have obtained the certificate;

Long-term mechanism: start from improving the product innovation ability of technical personnel design and engineering and technical problems, and promote the training and promotion of "1 +6 " regular and irregular innovative methods. Enterprise technical personnel should participate in the training with questions.

\section{References}

1. The Economic Significance of Patent Indicators and Its Practical Application [J]. Mao Hao. Intellectual property rights.

2. Takes listed companies listed on the Growth Enterprise Board as an example [J]. Zhang Xiaoyue, Chen Penglong, Zhao Weicheng.Research on science and technology management. 
3. high-quality patents push China towards a new stage of high-quality development [J]. Liu Huifeng. Science and technology China.

4. The Impact of [4] Patent Grant Policy on Patent Quality in China [J].Liu Xuefeng, Qin Lichao, Zhang Xiao.Intelligence exploration.

5. China Provincial Patent Funding Policy and Its Effect Analysis [D] Ma Yanhua.University of Science and Technology of China.

6. Research on inertial/visual navigation with headingposition constraints and the observability analysis[A]. Yujie Wang,Qingyang Chen,Peng Wang,Yafei Lu,Gaowei Jia.Proceedings of 5th International Conference on Automation, Control and Robotics Engineering (CACRE 2020) [C]. 2020 